\title{
PASSAGES DE TÉMOINS
}

\section{Claire Zalc}

Presses de Sciences Po | «Vingtième Siècle. Revue d'histoire »

$2018 / 3 \mathrm{~N}^{\circ} 139$ | pages 2 à 21

ISSN 0294-1759

ISBN 9782724635768

Article disponible en ligne à l'adresse :

https://www.cairn.info/revue-vingtieme-siecle-revue-d-histoire-2018-3-page-2.htm

Distribution électronique Cairn.info pour Presses de Sciences Po.

(C) Presses de Sciences Po. Tous droits réservés pour tous pays.

La reproduction ou représentation de cet article, notamment par photocopie, n'est autorisée que dans les limites des conditions générales d'utilisation du site ou, le cas échéant, des conditions générales de la licence souscrite par votre établissement. Toute autre reproduction ou représentation, en tout ou partie, sous quelque forme et de quelque manière que ce soit, est interdite sauf accord préalable et écrit de l'éditeur, en dehors des cas prévus par la législation en vigueur en France. Il est précisé que son stockage dans une base de données est également interdit. 
SPÉCIAL

L'histoire

de la Shoah

face à ses sources 


\title{
Passages de témoins
}

\author{
Claire Zalc
}

«Et quand la dernière survivante aura rejoint les siens dans le ciel de Pologne, nous laissant seuls avec pour héritage sa chancelante mémoire, qu'en ferons-nous, nous, les orphelins?»

(Jean-Claude Grumberg, Votre maman, 2012)

Le 4 janvier 2018, l'annonce du décès d'Aron Applefeld est venue rappeler, s'il était besoin, que les survivants de la Shoah ne seraient bientôt plus là pour témoigner. La disparition des témoins engendre-t-elle une modification dans les manières de penser, de concevoir, d'écrire, de se positionner face à l'histoire de la Shoah ${ }^{1}$ ? Cette interrogation hante une partie de la production artistique de ces dernières années, des Habitants de l'hôtel de Saint-Aignan en 1939, installation de Christian Boltanski sise dans la cour du Musée d'art et d'histoire du judaïsme, aux Disparus de Daniel Mendelsohn ${ }^{2}$. Qu'en est-il du côté des historiens ? Ce numéro entreprend d'aborder les transformations heuristiques, empiriques et théoriques engendrées par ce changement d'âge, à la fois historiographique et biographique. Peut-on parler de la fin de l'« ère du témoin », selon la terminologie inventée par Annette Wieviorka pour qualifier la période qui s'ouvre dans les années $1990^{3}$. Rien n'est moins sûr. La vague testimoniale ne tient pas nécessairement à la présence d'individus susceptibles de témoigner : elle a d'ailleurs marqué, pendant les années 1990, l'écriture de l'histoire d'autres génocides, comme celui des Arméniens. Le recours aux témoignages des victimes des massacres et des déportations s'impose à cette période comme l'un des moyens privilégiés de faire « comprendre et expliquer l'expérience de l'anéantissement ${ }^{4} »$. Dès lors, comment qualifier les changements qui semblent poindre, aujourd'hui, dans l'écriture de cette histoire ? Faut-il y voir un effet des modifications des regards historiens sur leurs matériaux ? Il semble que nous assistions, depuis quelques années, à une transformation de la relation entre les historiens et historiennes

(I) J'emploierai ici le nom donné à l'événement en France et en Israël qui tend à s'imposer aujourd'hui, même si c'est le terme Holocauste qui a été d'abord privilégié dans le monde anglo-saxon comme dans les organisations internationales : Annette Wieviorka, « Comprendre, témoigner, écrire », in Ivan Jablonka et Annette Wieviorka (dir.), Nouvelles Perspectives sur la Shoah, Paris, PUF, 2013, p. 5-18, p. 6 ; Tal Bruttmann et Christophe Tarricone, Les 100 Mots de la Shoah, Paris, PUF, « Que sais-je ? », 2016.

(2) Les articles de la rubrique «Images, lettres et sons » reviennent sur quelques productions artistiques récentes traitant du thème de la disparition des témoins.

(3) Annette Wieviorka, L'Ère du témoin, Paris, Plon, 1998.

(4) Alexandra Garbarini et Boris Adjemian, «Les violences de masse, de l'expérience au savoir », Études arméniennes contemporaines, 5, 2015, p. 7-13. 
et leurs sources. Le temps passe et éloigne, avec lui, les générations de l'événement : ce dernier ne concerne plus leurs parents, mais leurs grands-parents ${ }^{1}$. Dès lors, la nature des documentations sollicitées, leurs utilisations comme les rapports aux sources évoluent. Est-ce un effet de la disparition des derniers acteurs de cette histoire? Ou le corollaire d'un changement d'âge historiographique ? Ou s'agit-il plutôt d'un passage de témoin entre générations ?

Comme toute historiographie, celle de la Shoah est marquée par différentes strates, qui correspondent à différents temps et modes d'écritures de l'histoire qu'il n'est pas évident de résumer d'un trait, tant les évolutions correspondent au produit de multiples facteurs ${ }^{2}$. La terminologie même d'« histoire de la Shoah » n'est pas sans susciter des interrogations : le champ scientifique s'est progressivement institutionnalisé depuis une vingtaine d'années, à la faveur de la mise en place de centres de recherches internationaux, d'instances de financements dédiées, de revues spécialisées, de listes de diffusion ou encore d'associations professionnelles et de conférences internationales. Néanmoins, il reste difficile de délimiter précisément ce qui fait partie, ou non, de la Shoah, entre l'histoire des persécutions antisémites, de la diffusion de l'idéologie antisémite, des différentes modalités de mise en œuvre de la destruction des juifs ${ }^{3}$ d'Europe comme $^{\prime}$ de sa contention ${ }^{4}$, ou encore des acteurs variés du processus, dont l'acception est sans doute nettement plus complexe que ne le laisse croire la triade formalisée par Raul Hilberg distinguant bourreaux-victimes et témoins (bystanders).

Loin de proposer une définition exclusive ou inclusive de «l'histoire de la Shoah », ce numéro entend mener l'enquête en s'ouvrant à différents auteurs et exemples de recherches qui attestent de nouvelles sources ou, plus exactement, de nouveaux usages de sources, permis, engendrés ou encore suscités par la disparition des témoins de cette histoire, afin de sonder leur portée sur la production des savoirs sur la Shoah. S'interroger sur la spécificité de ce moment revient à prendre la mesure du «tournant testimonial » dans la pratique historienne. Ce tournant se situe au carrefour de plusieurs temporalités politiques, archivistiques, historiographiques et biographiques. De plus, s'il s'accompagne de l'identification de massifs documentaires, il tient aussi à la mise en valeur de nouveaux regards et manières de localiser, de lire et d'interpréter les sources. C'est pourquoi, afin de rendre compte des effets de la perte des témoins sur l'écriture du récit historien, ce numéro fait le choix de privilégier une focale microhistorienne, mais également de donner la parole à une nouvelle génération

(I) Ivan Jablonka, Histoire des grands-parents que je n'ai pas eus, Paris, Éd. du Seuil, 2011.

(2) Dan Michman, Pour une historiographie de la Shoab : conceptualisations, terminologie, définitions et problèmes fondamentaux, Paris, In Press éditions, 2001.

(3) Dans ce numéro spécial, le substantif « juif » est écrit avec une minuscule initiale, qu'il renvoie à un peuple ou à une religion.

(4) Sur ce point, voir Jacques Sémelin, Claire Andrieu et Sarah Gensburger (dir.), La Résistance au Génocide: de la pluralité des actes de sauvetage, Paris, Presses de Sciences Po, 2008. 
de chercheurs et chercheuses. En s'attelant à cette histoire, ils sont confrontés à trois difficultés majeures : se situer dans un paysage historiographique à la fois foisonnant, structurant et presque intimidant, travailler sur un événement dont l'histoire du $20^{\mathrm{e}}$ siècle a acté tant l'unicité que la centralité et se positionner face à une demande mémorielle et institutionnelle en plein essor depuis les années 1990. Dès lors, donner à voir comment se jouent les nouvelles relations des historiens et historiennes de la Shoah (si tant est que cette expression ait encore un sens) à leurs sources conduit à esquisser des pistes de réflexions sur certaines réponses apportées par cette génération, ainsi que sur les nouvelles approches d'un champ, appelé désormais à sortir de sa spécificité pour dialoguer avec d'autres, en bref à se décloisonner.

\section{Contextes politiques}

La relation aux sources est intimement liée à la combinaison de plusieurs temporalités : une temporalité politique tout d'abord, scandée dans chaque cadre national par des rythmes propres, notamment judiciaires et mémoriels ${ }^{1}$. On assiste en effet, depuis une trentaine d'années, à un mouvement d'accessibilité croissante d'un ensemble de fonds archivistiques jusque-là confinés loin du regard des chercheurs. Les changements politiques internationaux, avec la fin de la guerre froide et des régimes communistes en Europe, ont permis d'impressionnantes ouvertures de fonds d'archives ${ }^{2}$. Ces dernières ont engendré l'identification et l'exploitation de nombreux massifs documentaires, ce dont témoigne la vitalité des travaux sur l'Europe de l'Est, notamment sur la Pologne ou la Russie ${ }^{3}$. Ces ouvertures ne concernent d'ailleurs pas uniquement les pays de l'ancien Bloc soviétique puisqu'elles ont également permis le transfert de documents autrefois pillés à la faveur du second conflit mondial ${ }^{4}$. Dès le milieu des années 1990, face à l'abondance de la documentation mise au jour, le Centre de documentation juive contemporaine (CDJC) à Paris décide d'organiser une rencontre entre les historiens de la Shoah et les archivistes des principaux pays d'Europe afin d'en

(I) Sophie Cœuré, «Archives dans les guerres, guerres des archives aux Xx et $\mathrm{XXI}^{\mathrm{e}}$ siècles », Pouvoirs, 153, 2015 , p. 25-36.

(2) Sabine Dullin, « Le secret et ses usages : les archives "sensibles" en URSS », in Sébastien Laurent (dir.), Archives « secrètes », secrets d'archives? Historiens et archivistes face aux archives sensibles, Paris, CNRS éditions, 2003, p. 189-200 ; Sonia Combe (dir.), Archives et histoire dans les sociétés postcommunistes, Paris, La Découverte, 2009.

(3) Parmi quelques exemples récents, voir Jan Grabowski, Hunt for the fews: Betrayal and Murder in GermanOccupied Poland, Bloomington, Indiana University Press, 2013 ; Omer Bartov, Anatomy of a Genocide : The Life and Death of a Town Called Buczacz, Providence, Simon \& Schuster, 2018 ; Nathalie Moine, « La commission d'enquête soviétique sur les crimes de guerre nazis : entre reconquête du territoire, écriture du récit de la guerre et usages justiciers », Le Mouvement social, 222 (1), 2008, p. 81-109; Nathalie Moine et John Angell, « Defining "War Crimes against Humanity" in the Soviet Union », Cabiers du monde russe, 52 (2-3), 2011, p. 441-473.

(4) Sophie Cœuré, La Mémoire spoliée : les archives des Français, butin de guerre nazi puis soviétique (de 1940 à nos jours), Paris, Payot, 2007. 
penser les principaux apports. Un guide est alors publié pour coordonner les différentes institutions d'archives, localisées dans vingt-cinq pays européens ${ }^{1}$.

Ce contexte international se conjugue à certaines dynamiques nationales. Prenons l'exemple de la France; à la suite du discours du président Jacques Chirac le 16 juillet 1995 à l'occasion de la commémoration de la rafle du Vél' d'hiv', du travail de la commission Mattéoli puis de la mobilisation de nombreux historiens, menée notamment par Gilles Morin et Sophie Coeuré au printemps 2015, l'arrêté ministériel du 24 décembre 2015 «portant ouverture d'archives relatives à la Seconde Guerre mondiale » promulgue une « dérogation générale destinée à faciliter l'accès aux archives publiques relatives à la Seconde Guerre mondiale ».

La France ne constitue pas un cas isolé. Il suffit de quelques exemples récents pour rappeler que ce mouvement d'ouverture et de facilitation d'accès ne cesse de progresser, depuis le début du $21^{\mathrm{e}}$ siècle, non sans quelques à-coups. Il n'obéit pas, loin s'en faut, à un rythme linéaire et continu, et reste là aussi, bien souvent, déterminé par la mobilisation individuelle ou collective d'historiens, de chercheurs ou de personnalités.

\section{Contextes archivistiques}

Les initiatives de collecte de sources ont pris un nouvel essor dans les années 1980, sous l'impulsion de politiques mémorielles et de transformations technologiques. La campagne de recueil d'archives filmées de témoignages de survivants, lancée en 1979 à New Haven est déposée à l'Université de Yale en 1981 sous le nom de Fortunoff Video Archive for Holocaust Testimonies. D'une part, elle résulte d'une politisation de la mémoire de l'Holocauste aux États-Unis, avec la mise en place de la commission sur l'Holocauste par le président Carter en 1978, de l'investissement personnel de Geoffrey Hartman, professeur de littérature comparée, et de Dori Laub, psychanalyste et lui-même survivant de la Shoah. D'autre part, elle est largement accélérée par la diffusion de caméras vidéo légères et accessibles. La révolution scientifique des années 1990 modifie ainsi, en profondeur, les politiques archivistiques, muséographiques et patrimoniales. Si le photocopiage, puis le microfilmage avaient déjà joué un rôle précurseur en la matière, la numérisation permet de démultiplier les points d'accessibilité de certains fonds. Cette temporalité technologique déploie les possibilités de consultation à distance et de reproduction des sources ${ }^{2}$. De même, certaines institutions mémorielles, comme l'United States Holocaust Memorial Museum (USHMM) ou l'Institut international Yad Vashem pour la mémoire de

\footnotetext{
(I) Guide européen des sources d'archives sur la Shoah, Paris, CDJC, 1999.

(2) Yann Potin, « Institutions et pratiques d'archives face à la "numérisation" : expériences et malentendus », Revue d'bistoire moderne et contemporaine, 58 (4 bis), 2011, p. 57-69.
} 
l'Holocauste en Israël, sont devenues des lieux incontournables de la recherche sur la Shoah. Ils ont constitué des collections de fonds provenant de dizaines de pays différents qui ne sont pas conservées en propre dans leurs locaux, mais consultables sur place grâce à la production en masse de copies, microfilmées ou numérisées. Il est ainsi désormais possible de consulter dans un même lieu des fonds auparavant éparpillés de par le monde. Depuis mai 2015, l'European Holocaust Research Infrastructure (EHRI) tente de réunir, cette fois-ci virtuellement uniquement, un ensemble d'informations relatives aux sources de la Shoah, dispersées sur le territoire européen ${ }^{1}$.

Devant la nécessité de considérer les sources non comme un donné du savoir historique mais comme le produit de pratiques sociales, politiques, muséales et mémorielles, la rubrique «Archives » de ce numéro, particulièrement fournie pour l'occasion, présente l'histoire de la constitution de fonds documentaires qui viennent renouveler en profondeur les approches. La collection de l'International Tracing Service (ITS), d'une part, à la fois considérable et particulièrement difficile à utiliser du fait de sa composition disparate, est présentée par Diane F. Afoumado; Constance Pâris de Bollardière revient sur les différentes strates de collections de sources audiovisuelles rassemblant des témoignages de victimes, qui offrent de larges possibilités d'exploitation comme savoirs autant sur l'événement que sur la mémoire de celui-ci². La compréhension de l'histoire de ces sources éclaire leurs usages possibles, ce qu'illustre la contribution de Laurence Schram. Elle décrit en effet les potentialités des croisements d'archives nés de l'histoire d'un lieu, la caserne Dossin à Malines, rouage essentiel de la Shoah en Belgique qui a rassemblé de 1942 à 1944, 25000 juifs et 350 Tsiganes, avant leur déportation à Auschwitz-Birkenau.

\section{Contextes historiographiques}

Il serait naïf de penser que les transformations de l'histoire de la Shoah ne sont portées que par la collecte, la mise à disposition, la découverte ou l'exploitation de nouveaux fonds. Elles sont également impulsées par une temporalité proprement historiographique. On assiste en effet, ces dix dernières années, à un renouveau des approches dans un cadre à la fois microhistorien et transnational. L'historiographie de la Shoah est aujourd'hui abondante et son cadre général, celui des étapes de la destruction des juifs d'Europe, solidement établi, depuis que les études pionnières de Léon Poliakov et de Gerald Reitlinger ${ }^{3}$ en ont jeté

(I) https ://www.ehri-project.eu.

(2) Pour un usage exemplaire des témoignages recueillis ex post, voir Christopher Browning, À l'intérieur d'un camp de travail nazi. Récits des survivants : mémoire et histoire, Paris, Les Belles Lettres, 2010.

(3) Léon Poliakov, Bréviaire de la haine: le III Reich et les fuifs, Paris, Calmann-Lévy, 1951 ; Gerald Reitlinger, The Final Solution: The Attempt to Exterminate the fews of Europe, 1939-1945, Londres, Vallentine Mitchell, 1953. 
les premières bases au début des années 1950, suivies par les travaux de Raul Hilberg puis ceux de Saul Friedländer ${ }^{1}$. Dans les années 1980 et 1990, des travaux menés sur un espace plus restreint, bien souvent local, ont permis de mieux appréhender les mécanismes de la mise en place de la Solution finale, comme par exemple dans le district de Lublin, de Riga ou de la Biélorussie ${ }^{2}$. Cette focale resserrée conduit à comprendre et à éclairer un ensemble bien plus vaste ${ }^{3}$. Depuis plusieurs années, c'est au sein d'une démarche qui se revendique plus directement de la microhistoire que se sont multipliés des travaux portant sur des segments particuliers de l'histoire de la Shoah, comme sur un camp, un ghetto, une rafle, une ville, un convoi, une famille ou encore un bataillon ${ }^{4}$. En 2008, Saul Friedländer rappelait l'importance d'une étude des interactions entre les juifs de l'Europe allemande et les forces contribuant à la mise en œuvre de la Solution finale : «C'est à ce microniveau qu'il est le plus nécessaire de l'étudier ${ }^{5}$. »

L'observation à la loupe de la destruction des juifs d'Europe permet d'éclairer les relations d'interconnaissance entre victimes et bourreaux, de replacer les comportements individuels dans leurs environnements sociaux et, ce faisant, de comprendre le processus de mise à mort en l'insérant dans des configurations locales ${ }^{6}$. Cependant, ce tournant historiographique doit être resitué dans sa complexité, qui ne se restreint pas à la réduction de la focale d'observation ${ }^{7}$.

(1) Raul Hilberg, La Destruction des Zuifs d'Europe, Paris, Fayard, 1961, 1988 ; Saul Friedländer, Les Années d'extermination : l'Allemagne nazie et les fuifs, 1939-1945, Paris, Ed. du Seuil, 2008.

(2) Dieter Pohl, Von der «Fudenpolitik» zum fudenmord: der Distrikt Lublin des Generalgouvernements, 1939-1944, Berne, Peter Lang, 1993 ; Christian Gerlach, Kalkulierte Morde: die deutsche Wirtschafts- und Vernichtungspolitik in Weissrussland 1941 bis 1944, Hambourg, Hamburger Edition, 1999; Andrej Angrick et Peter Klein, The « Final Solution » in Riga : Exploitation and Annibilation, 1941-1944, New York, Berghahn Books, 2009.

(3) Sur l'apport de la démarche monographique à l'analyse du nazisme, voir l'ouvrage classique de William S. Allen, The Nazi Seizure of Power: The Experience of a Single German Town, 1930-1935, Chicago, Quadrangle Books, 1965 ; trad. fr., id., Une petite ville nazie : 1930-1935, préf. d'Alfred Grosset, trad. de l'angl. par Renée Rosenthal, Paris, Robert Laffont, 1967, 2016 ; Henry Huttenbach, The Destruction of the Jewish Community of Worms, 1933-1945: A Study of the Holocaust Experience in Germany, New York, Memorial Committee of Jewish Victims of Nazism from Worms, 1981 ; Panikos Panayi, « Victims, Perpetrators and Bysbanders in a German Town : The Jews of Osnabrück before, during and after the Third Reich », European History Quarterly, 33 (4), 2003, p. 451-492.

(4) Pour n'en citer que quelques-uns, mentionnons Christopher Browning, Ordinary Men : Reserve Police Battalion 101 and the Final Solution in Poland, New York, Harper Collins, 1992, trad. fr., id., Des hommes ordinaires : le 101 bataillon de réserve de la police allemande et la Solution finale en Pologne, préf. de Pierre Vidal-Naquet, trad. de l'angl. par Élie Barnavi, Paris, Les Belles Lettres, 1994, 2007 ; id., Remembering Survival : Inside a Nazi Slave-Labor Camp, New York, W. W. Norton, 2010, trad. fr., id., À l'intérieur d'un camp de travail nazi : récits des survivants, mémoire et histoire, trad. de l'angl. par Jacqueline Carnaud, Paris, Les Belles Lettres, 2010, Pluriel, 2013 ; Mark Roseman, A Past in Hiding : Memory and Survival in Nazi Germany, New York, Metropolitan Book, 2000 ; Götz Aly, Into the Tunnel: The Brief Life of Marion Samuel, 1931-1943, New York, Metropolitan Books, 2007 ; Omer Bartov, Anatomy of a Genocide: The Life and Death of a Town Called Buczacz, New York, Simon \& Schuster, 2018.

(5) S. Friedlander, Les Années d'extermination, op. cit., p. 26.

(6) Voir les études réunies dans Claire Zalc, Tal Bruttmann, Ivan Ermakoff et Nicolas Mariot (dir.), Pour une microbistoire de la Shoah, Paris, Éd. du Seuil, « Le genre humain », 2012 ; et dans Claire Zalc et Tal Bruttmann (dir.), Microhistories of the Holocaust, New York, Berghahn Books, 2016.

(7) Certains travaux optent ainsi pour une échelle mésoscopique : Nicolas Mariot et Claire Zalc, Face à la persécution : 991 Juifs dans la guerre, Paris, Odile Jacob, 2010 ; Evgeny Finkel, Ordinary fews : Choice and Survival during the Holocaust, Princeton, Princeton University Press, 2017. 
En effet, il convient de distinguer les approches microhistoriennes des études monographiques ${ }^{1}$. Comme Ivan Ermakoff le démontre dans son article, l'approche microhistorique revient à faire cas de l'étude d'un cas. En ce sens, la microhistoire se différencie de l'histoire locale, puisque la démarche restitue l'espace des possibles en mettant l'accent sur la distorsion entre le cas et le général : c'est ainsi que Edoardo Grendi évoque « l'exceptionnel normal », qui conduit à se déprendre des questions de représentativité ${ }^{2}$. Mettre l'accent sur les « espaces des possibles » conduit à centrer les propos sur les stratégies des acteurs et les marges de manœuvre disponibles, souvent subsumés dans la notion anglosaxonne d'agency.

En ce sens, le geste microhistorique suscite la mobilisation de regards spécifiques sur les matériaux historiens, interrogés comme indices d'actions possibles. C'est pourquoi qu'il a paru intéressant de donner à voir, dans ce numéro, essentiellement des recherches qui avaient recours à la focale micro. Les tentatives de reconstruction microhistoriques procèdent d'indice(s), comme le rappelle Carlo Ginzburg qui décrit cette mécanique d'un savoir procédant à partir du cumul de traces, comme une chasse :

Pendant des millénaires, l'homme a été un chasseur. Au cours de ses innombrables chasses, il a appris à reconstituer les formes et les déplacements de proies invisibles à partir d'empreintes laissées dans la boue, de branches cassées, d'excréments, de touffes de poils, de plumes arrachées, d'odeurs confinées. [...] De la même façon, c'est en recueillant les traces et les indices les plus infimes du passé que l'on est habilité à élaborer une histoire précise de l'humanité, à la façon des archéologues, des paléontologues et des graphologues qui font l'histoire en partant du détail ${ }^{3}$.

Il est troublant de rappeler que ces compétences cynégétiques constituent, selon Christian Ingrao, l'une des caractéristiques des agissements de l'unité SS dite brigade Dirlewanger dans sa lutte contre les partisans soviétiques en Biélorussie ${ }^{4}$. Ainsi, l'histoire de la Shoah n'échappe pas aux tendances lourdes qui marquent l'historiographie.

Au-delà de la chasse aux indices, portée par la démarche microhistorienne, l'ouverture transnationale constitue d'ailleurs un horizon encore balbutiant, mais sans conteste appelé à se développer. C'est le sens des travaux centrés sur les organisations internationales d'aide aux survivants ou encore des recherches prosopographiques sur les trajectoires transnationales de victimes de la persécution ${ }^{5}$.

(I) La confusion tient aussi à des acceptions nationales différentes de ce qu'on nomme « microhistoire ». Voir Francesca Trivellato, «Microstoria/Microhistoire/Microhistory », French Politics, Culture, and Society, 33 (1), 2015, p. 122-134.

(2) Edoardo Grendi, « Micro-analisi e storia sociale », Quaderni storici, 35 (2), 1977, p. 506-520.

(3) Ginzburg Carlo, « "Signes, traces, pistes” : racines d'un paradigme de l'indice », Le Débat, 6, 1980, p. 9-10.

(4) Christian Ingrao, Les Chasseurs noirs : la brigade Dirlewanger, Paris, Perrin, 2006.

(5) Dalia Ofer, Françoise S. Ouzan et Judy Tydor Baumel-Schwartz (dir.), Holocaust Survivors : Resettlement, Memories, Identities, New York, Berghahn Books, 2011 ; Nicolas Mariot et Claire Zalc, « Reconstructing 
La difficulté de ces recherches tient non seulement aux compétences polyglottes requises, mais aussi à la maîtrise de contextes nationaux extrêmement variés ${ }^{1}$. On le voit, la temporalité historiographique contribue à modifier le type et la nature des sources exploitées, mais également les relations des individus qui écrivent cette histoire à leurs objets-sujets.

\section{Le temps des disparus}

Enfin, il convient d'adjoindre une dernière temporalité qu'on peut qualifier de biographique. Les femmes et les hommes ayant vécu cette histoire ont longtemps été partie prenante centrale de la construction de l'historiographie : bourreaux, mis en cause dans des cadres judiciaires ; victimes appelées à témoigner dans différents cadres; témoins (bystanders), interpellés sur leurs silences, leur participation, leurs regards ; « justes », oubliés, reconnus ou décorés. Or, dans quelque temps, ces personnes ne seront plus là pour intervenir.

La disparition des survivants vient alimenter des renouvellements divers : la publication de séries de sources, à l'instar des volumes de documents sur la persécution et le meurtre des juifs en Europe par l'Allemagne nazie, ou encore de la collection intitulée « Documenting Life and Destruction » publiée par le Center for Advanced Holocaust Studies de l'USHMM ${ }^{2}$. Hors du monde académique, la parution d'un ensemble de journaux intimes de « bourreaux » comme de « victimes » pour reprendre la terminologie de Raul Hilberg, les campagnes de collecte systématique de témoignages dans des formes diverses (écrites, enregistrées et/ou filmées), l'utilisation de nouveaux matériaux (images, films, lieux ou objets) contribuent à modifier les regards sur l'histoire de la Shoah, en lien avec la disparition des acteurs de cette histoire. Comment se reconfigure le rapport entre histoire, mémoire et politique alors que les survivants meurent, que les enfants des survivants vieillissent, que l'antisémitisme se déploie sous de nouvelles formes ${ }^{3}$ ? Est-il possible de penser les effets de la disparition prochaine des acteurs de cette histoire sur l'accessibilité, le recours, mais également les lectures des sources de la Shoah ? Il est à parier qu'elle rende les historiens et historiennes un peu plus aptes à se déprendre, voire à se libérer d'une histoire assimilée à la « reconstruction/résurrection du passé ${ }^{4} \gg$. Plus libres, mais orphelins. L'enjeu consiste aussi

Trajectories of Persecution : Reflections on a Prosopography of Holocaust Victims », in C. Zalc et T. Bruttmann (dir.), Microbistories..., op. cit., p. 85-112 ; Eric T. Jennings, Escape from Vichy: The Refugee Exodus to the French Caribbean, Harvard, Harvard University Press, 2018.

(I) Christian Gerlach, The Extermination of the European fews, Cambridge, Cambridge University Press, 2016.

(2) Susanne Heim et al. (dir.), Die Verfolgung und Ermordung der europäischen fuden durch das nationalsozialistische Deutschland, 1933-1945, Munich, Oldenbourg, 2008 à 2017, 14 vol., deux volumes supplémentaires à paraitre ; Jürgen Matthaus et al. (dir.), Documenting Life and Destruction: Holocaust Sources in Context, Lanham, AltaMira Press, 2010-2016, 11 vol.

(3) Samuel Ghiles-Meilhac, « Mesurer l'antisémitisme contemporain : enjeux politiques et méthode scientifique », Revue d'histoire moderne et contemporaine, 62 (2-3), 2015, p. 201-224.

(4) Joseph Morsel, « Traces ? Quelles traces ? Réflexions pour une histoire non passéiste », Revue historique, 680, 2016, p. 813-868, p. 848. 
à interroger, au sens propre comme au sens figuré, les modalités du passage de témoin entre générations historiennes.

\section{De la découverte d'un continent à l'exploration d'un espace}

Il est incontestable que l'œuvre des pionniers de l'historiographie de la Shoah est inextricablement liée à celle de localisation et d'identification de sources, voire parfois même de collecte. Léon Poliakov se charge de recueillir, pour le Centre de documentation juive contemporaine, les preuves documentaires de l'extermination des juifs avant de faire paraître le Bréviaire de la haine, en 1951. Itzhok Niborski et Annette Wieviorka identifient dès le début des années 1980 l'importance des livres du souvenir ${ }^{1}$. Dans un ouvrage publié en 2000, Raul Hilberg se livre à un panorama introspectif de la question des sources de la Shoah. Après la mise au jour de faits et l'écriture du récit, il lui semble nécessaire de livrer sa réflexion sur les sources de cette histoire ${ }^{2}$. Celle-ci est sans conteste le produit de sa trajectoire : pionnier de cette historiographie, Raul Hilberg offre une lecture quelque peu galiléenne d'un corpus de sources, formant une sorte de continent qu'il a fallu explorer, parcourir, défricher et exploiter, à l'instar des territoires pénétrés par de premières expéditions d'aventuriers, puis peu à peu apprivoisés. Pour lui, les « archives documentaires se sont peu à peu rétrécies, jusqu'au moment où il n'en est plus resté qu'un ensemble fini ${ }^{3} \gg$.

Or, au-delà de la finitude du continent à découvrir, l'observation des recherches qui se déploient aujourd'hui dans le champ des études de la Shoah laisse à penser qu'elles s'inscrivent au sein d'un voyage à travers l'espace, infini, d'identifications de sources et d'invention de regards portés sur celles-ci. Les réflexions initiées par les historiens et les historiennes des périodes plus anciennes, les médiévistes notamment, ont conduit à questionner la notion même de « disponibilité des sources ${ }^{4} \gg$. Ce n'est pas l'impossibilité d'accéder aux objets d'études qui définit le métier d'historien comme « connaissance par traces », pour reprendre l'expression consacrée, mais bien une épistémologie à part entière d'une discipline dont la relation au réel est médiée par la source ${ }^{5}$. Or l'historiographie de la Shoah s'est longtemps définie à l'écart de cette définition générique. Dans sa réflexion générale sur les relations entre mémoire et histoire, Paul Ricœur fait ainsi exception des témoignages des rescapés de la Shoah, qu'il qualifie, à la suite de Saul Friedländer, d'« expériences à la limite »: «l'expérience à transmettre est celle

(I) Annette Wieviorka et Itzkhok Niborski, Les Livres du souvenir : mémoriaux juifs de Pologne, Paris, Gallimard/ Julliard, 1983.

(2) Raul Hilberg, Les Sources de la Shoah, Paris Gallimard, 2000.

(3) Ibid., p. 22.

(4) Yann Potin, « Archiver l'enquête ? Avatars archivistiques d'un monument historiographique : les enquêtes “administratives" de Louis IX (1247-1248) », in Claude Gauvard (dir.), L'Enquête au Moyen Âge, Rome, École française de Rome, 2008, p. 241-267 ; Joseph Morsel, «Les sources sont-elles "le pain de l'historien" ? », Hypothèses, 7 (1), 2004, p. 271-286.

(5) J. Morsel, « Traces? Quelles traces ? », art. cité. 
d'une inhumanité sans commune mesure avec l'expérience de l'homme ordinaire $»^{1}$. La période fut longtemps marquée du sceau de l'exceptionnalité, de par sa proximité avec un événement qualifié d'indicible et d'impensable. Ce statut a engendré un rapport particulier aux traces marqué par l'obsession de vérité et de preuves d'abord, cela afin de faire pièce tant à la volonté nazie d'étouffer le meurtre par l'effacement des traces qu'aux thèses négationnistes qui se diffusent dans l'après-guerre ${ }^{2}$. Il semble que l'on assiste aujourd'hui à une prise de distance vis-à-vis de ces postures militantes pour retrouver une relation plus habituelles aux sources de l'histoire.

Dès lors qu'il s'agit moins de faire preuve que de chercher du sens, le triptyque trace, document, question, «trépied de base de la connaissance historique ${ }^{3}$ », laisse la porte ouverte aux renouvellements, sans doute infinis, des écritures de l'histoire, puisque les interrogations posées au passé se multiplient au rythme du présent. Pendant des années, les récits se sont élaborés en présence, physique ou mentale, des acteurs; les historiens et historiennes ont été notablement pris à partie dans les arènes judiciaires et mémorielles ; les histoires ont été écrites et pensées dans le contexte militant et politisé de la lutte contre le négationnisme. Certes, les combats n'ont pas fini d'être menés : l'inquiétante actualité nous le rappelle alors que la Pologne vient d'adopter une loi menaçant de trois ans de prison toute personne polonaise ou étrangère qui «attribue à la république de Pologne et à la nation polonaise publiquement et contre la réalité des faits, la responsabilité ou la coresponsabilité de crimes nazis perpétrés par le Troisième Reich allemand ${ }^{4} \gg$. Les contextes ne sont pas apaisés : les débats restent vigoureux et les controverses aussi. Cependant, les individus qui ont vécu cette histoire ne seront très prochainement plus là. Dès lors, ce numéro entend simplement produire un état des lieux des relations dialogiques des historiens et historiennes aux sources de la Shoah, en les considérant comme des pratiques sociales, nécessairement historicisées. En ce sens, l'expérience d'écriture de l'histoire du génocide au Rwanda, depuis une vingtaine d'années, a sans conteste conduit à démarquer l'historiographie de la Shoah de la problématique moins de l'exceptionnalité que de l'unicité 5 . Pour autant, la fin de la confrontation avec les témoins débarrasse-t-elle, comme en appelle Étienne Anheim, des « problématiques de la mort, de la dette et de la sépulture » chères à Paul Ricœur ${ }^{6}$ ? Ce

(I) Paul Ricœur, La Mémoire, l'histoire, l'oubli, Paris, Éd. du Seuil, 2000, p. 223 ; Saul Friedländer, Probing the Limits of Representation : Nazism and the «Final Solution », Cambridge, Harvard University Press, 1992.

(2) Sur la France, voir Valérie Igounet, Histoire du négationnisme en France, Paris, Éd. du Seuil, 2000.

(3) P. Ricœur, La Mémoire..., op. cit., p. 226.

(4) Jean-Charles Szurek, « L'amère victoire des historiens polonais », Libération, 20 février 2018.

(5) Même si toute comparaison soulève, encore, d'intenses polémiques, ce dont témoignent les réactions à la publication de l'ouvrage de Sidi N'Diaye, Tutsis du Rwanda et fuifs de Pologne victimes de la même baine ?, Paris, Le Bord de l'eau, 2017. Voir le compte rendu page 219 de ce numéro.

(6) Étienne Anheim, «Singulières archives : le statut des archives dans l'épistémologie historique. Une discussion de La Mémoire, l'histoire, l'oubli de Paul Ricœur », Revue de synthèse, 125, 2004, p. 153-182, p. 180. 
numéro ne prétend aucunement proposer quelque inventaire exhaustif de ces approches ; il donne à voir quelques exemples à même d'illustrer les évolutions en cours afin de penser la nature et la direction des changements à l'œuvre.

\section{Élargir les sources, élargir le champ}

Le mouvement voit l'élargissement de la notion de sources à des matériaux comme la littérature, la photographie ou les films. Le choix est limité ; nous aurions pu inclure d'autres segments en plein essor comme l'archéologie des sites et des fosses ${ }^{1}$. Nous avons préféré, sans nous limiter au constat, largement partagé, de la nécessité d'étendre le spectre des ensembles documentaires, mener une réflexion sur les postures épistémologiques à défendre en regard de ces objets. Les relations de la littérature à la Shoah ont fait l'objet de récentes réflexions novatrices, de la poétique de la littérature de témoignages à l'étude du positionnement des écrivains face à la Shoah ${ }^{2}$.

La disparition des acteurs renouvelles les regards sur des documents habituels, voire iconiques de l'histoire de la Shoah à l'instar de l'« album d'Auschwitz » qui comporte des photographies des opérations de « sélection » sur la rampe située entre les deux parties du camp de Birkenau. Si les clichés sont mondialement connus, on ignore quasiment tout de l'histoire de cet album. Tal Bruttmann, Christoph Kreutzmüller et Stefan Hördler tentent de remédier à ce vide par une enquête serrée, au plus près de la fabrique des images, qui vient proposer des acquis novateurs quant à la fonction, aux destinataires et aux auteurs des photographies. Les interrogations sur l'objet photographique apportent un éclairage tout à fait pionnier sur l'histoire du camp d'Auschwitz et sur la mise en œuvre du processus d'extermination. Elles présentent notamment la topographie des lieux de la mise à mort, dont les auteurs montre avec brio combien les contemporains peinent à le comprendre.

Judith Lyon-Caen s'attache par ailleurs à penser les usages historiens des sources littéraires, en travaillant sur l'anthologie de poèmes en polonais publiée par Michel Borwicz, dans le cadre des travaux de la Commission centrale historique juive de Pologne. Elle montre l'intérêt d'une étude des modalités et des contextes de composition, d'écriture, de conservation, de diffusion et de parution de ces poèmes, considérés non comme des «sources » sur le vécu de leurs auteurs, mais comme des «actions d'écriture ». Pour ces survivants, les poèmes témoignent de la lutte contre la déshumanisation par le travail poétique

(I) Élisabeth Anstett et Jean-Marc Dreyfus (dir.), Cadavres impensables, cadavres impensés : approches méthodologiques du traitement des corps dans les violences de masse et les génocides, Paris, Petra, 2013 ; « Material Traces of Mass Death : The Exhumed Object/Traces matérielles de la mort de masse : l'objet exhumé », dossier dirigé par Sophie Baby et François-Xavier Nérard, Cabiers Sirice, 19, 2017.

(2) Catherine Coquio, La Littérature en suspens. Écritures de la Shoab: le témoignage et les xuvres, Paris, L'Arachnéen, 2015 ; Agnieszka Grudzińska, Victimes, témoins : les écrivains polonais face à la Shoah, 1940-1960, Paris, Classiques Garnier, 2015. 
et constituent un geste ultime de transmission. L'effet du contexte d'écriture est revendiqué comme moteur : c'est d'une certaine manière «parce que nous écrivons en un temps où, précisément, les derniers survivants disparaissent » qu'apparaît la valeur documentaire et testimoniale singulière de la littérature qui conduit Judith Lyon-Caen à voir « derrière chaque écrit, chaque décision d'écriture, chaque trace arrachée à la disparition, l'histoire d'une résistance et d'un combat ».

La source visuelle est également abordée à travers un ensemble de films du ghetto de Kaunas en Lituanie et du camp de Klooga en Estonie, tournés entre l'été et l'automne 1944. Irina Tcherneva conjugue analyse visuelle et histoire sociale pour rendre compte des usages de ces sources audiovisuelles. Elle étudie les conditions de tournage et les possibilités de filmage à la fin du conflit, la fabrique des regards sur le génocide, leur prise en charge institutionnelle, les modes de stockage mais aussi les destins opposés des captations : relégation et oubli dans un cas, exposition internationale dans l'autre. Considérer cette source conduit à montrer le poids des contraintes matérielles au moment de la découverte des camps et des crimes de masse, ainsi que les choix de focales et les images écartées ou absentes ${ }^{1}$.

L'élargissement tient également à l'identification de nouveaux objets de recherche. Ainsi Audrey Kichelewski étudie-t-elle des landsmanshaftn, ces sociétés d'originaires qui rassemblent des juifs venus de la même ville ou du même village, mais aussi appartenant au même groupe professionnel, et qui jouent le rôle de sociétés de secours mutuel. Elle relate un parcours semé d'embûches, ces archives privées disparaissant le plus souvent avec les dirigeants qui les conservaient à domicile. C'est donc au moyen de déplacements d'échelles qu'elle esquisse des pistes : enquêtes ethnographiques auprès des enfants, dépouillements des sources imprimées, analyses de corpus photographiques et de tombes collectives, financés par ces landsmanshaftn, dans les pays d'émigration. Les déplacements vont aussi dans le sens d'une intégration, dans l'historiographie, de corpus de sources venus d'autres champs, ne portant pas à première vue directement sur la Shoah. Le processus même d'extermination s'accompagnant d'une volonté de destruction systématique de toutes traces du meurtre, il a fallu dès les premiers temps chercher autour et à côté de l'événement. Raul Hilberg établissait déjà, en 2000 , que « les sources documentaires relatives à la catastrophe juive ne forment donc pas une section reconnaissable parmi les matériaux qui ont subsisté ${ }^{2} \gg$. Ces détours s'élargissent aujourd'hui à des documents qui a priori ne portent pas l'estampille « histoire de la Shoah », mais contribuent à en éclairer certains pans.

(I) Sur le cinéma français, voir Sylvie Lindenperg, Les Écrans de l'ombre : la Seconde Guerre mondiale dans le cinéma français (1944-1969), Paris, Éd. du Seuil, 1997.

(2) R. Hilberg, Les Sources de la Shoah..., op. cit., p. 34. 


\section{Scruter et croiser les egos}

Le mouvement se caractérise ensuite par un déplacement de l'acception de l'archive, qui conduit notamment à une attention marquée pour les sources dites de l'intime. Lintérêt des ego-documents pour l'histoire, démontrée dans d'autres contextes et pour d'autres périodes ${ }^{1}$, vient dans le cas de la Shoah à la fois suppléer la disparition des témoins en leur donnant une « voix », tout en bénéficiant d'une certaine liberté à la fois d'accès, de lecture et d'utilisation.

La proposition de Barbara Engelking est à cet égard passionnante : dans quelle mesure est-il possible de considérer les rêves comme sources? Son enquête, fondée sur cent trente-sept rêves relatés par une soixantaine de victimes polonaises de la Shoah dans leurs mémoires, carnets, lettres et journaux intimes, interroge l'utilité de ce matériau original pour rendre compte de l'expérience subjective de la persécution, du massacre des siens et de sa propre exclusion du monde des vivants. Au-delà du parti pris historiographique, résolument distinct d'une approche psychanalytique, cet article invite à la comparaison des subjectivités survivantes dans d'autres génocides. C'est pourquoi Hélène Dumas, historienne du génocide rwandais, introduit ce texte en explicitant en quoi il ouvre de fécondes pistes de discussions.

Du côté des «bourreaux », une attention particulière est portée également aux ego-documents, catégorie floue comprenant écrits privés, correspondances et journaux. Alors que l'historiographie fut longtemps clivée entre l'histoire des bourreaux et celle des victimes, la première fondée sur les archives administratives, la seconde utilisant les témoignages, les mouvements s'inversent, dans la lignée ouverte notamment par Christopher Browning ${ }^{2}$. Si l'analyse de la langue nazie a contribué à éclairer les univers référentiels des génocidaires, qu'en estil des sources du for privé 3 ? Nicolas Mariot entreprend de décrypter les notes quotidiennes rédigées par Johann Paul Kremer, médecin en poste à Auschwitz, entre le 30 août et le 18 novembre 1942. Porter attention aux « formules sibyllines » et aux ellipses, mais revenir aussi sur les différentes traductions de ces notes (et leurs interprétations par les historiens qui ont, successivement, cité et utilisé cette source) donne à voir en creux l'évolution de l'historiographie. C'est alors à la question des relectures de sources que cette analyse conduit, ou des « revisites de sources » pour emprunter à la stimulante proposition de revisite des enquêtes ethnographiques, encore si peu développée en histoire ${ }^{4}$. Dans ce cadre, les croisements de sources venues d'horizons divers s'avèrent féconds. Nicolas Mariot prend le parti d'adosser sa lecture du journal de Johann

(I) Voir ainsi «Histoire et archives de soi », numéro spécial de Sociétés et représentations, 13, avril 2002, et notamment Philippe Artières et Dominique Kalifa, « L'historien et les archives personnelles : pas à pas », p. 7-15.

(2) C. Browning, À l'intérieur d'un camp de travail nazi..., op. cit.

(3) Johann Chapoutot, La Loi du sang : penser et agir en nazi, Paris, Gallimard, 2015.

(4) Michael Burawoy, « Revisits : A Turn to Reflexive Anthropology », American Sociological Review, 68, 2003, p. 645-679. 
Paul Kremer aux archives de l'ITS, dont les fonds permettent de reconstituer, convoi après convoi, les arrivées à Auschwitz-Birkenau. La confrontation des deux sources l'amène à attester de « rencontres », de papier tout au moins, entre le médecin SS et certains déportés exterminés dans le camp.

Nicolas Patin démontre quant à lui l'intérêt historique des journaux des bourreaux, à partir de l'étude des écrits de deux hauts responsables nazis, FriedrichWilhelm Krüger (1894-1945) et Erich von dem Bach-Zelewski (1899-1972). Tout en récusant le qualificatif de «sources intimes », il démontre ce que ces pratiques diaristes apprennent de la relation des nazis au temps et de leur insertion générationnelle. Ces sources offrent également un matériau d'analyse de l'économie de l'euphémisation du crime.

\section{Paroles, silences et non-dits}

Ces nouvelles approches contribuent à relire, à nouveaux frais, la question du « que savait-on et quand », sujet d'intenses débats historiographiques ${ }^{1}$. La nécessité de se démarquer de tout anachronisme s'avère à la fois particulièrement essentielle et difficile lorsqu'il s'agit d'analyser les paroles, faits et gestes des acteurs de cette histoire.

Ainsi, l'on reste saisi, dans un premier temps, devant le constat froid que dresse Louis Sadosky, inspecteur des renseignements généraux français, le 20 juillet 1942, soit quelques jours après la rafle du Vél' d'hiv', quand il affirme que l'intention de Hitler est «la destruction complète et à jamais de la race [juive] ${ }^{2}$ ». Laurent Joly défend cependant que c'est l'organisation même du système de persécution (division des tâches, chevauchement des compétences, « externalisation » du crime) qui explique l'absence du sentiment de responsabilité. La confrontation entre les actes de Sadosky lors des rafles parisiennes de l'été 1942, ses écrits durant ce même été 1942 et ses dénis lors des procès d'épuration conduit Laurent Joly à trouver une cohérence interne dans les comportements de ce policier, qui tient à son ethos professionnel.

Elissa Maïlander passe au crible d'un faisceau de témoignages un événement singulier : la pendaison d'une détenue au camp de Madjanek en 1943. Elle mène une réflexion sur la diversité des expériences vécues et sur la polyphonie

(I) Bernward Dörner, Die Deutschen und der Holocaust: was niemand wissen wollte, aber jeder wissen konnte, Berlin, Propyläen Verlag, 2007 ; Peter Longerich, «Davon haben wir nichts gewusst! 》 Die Deutschen und die Fudenverfolgung, 1933-1945, Munich, Siedler Verlag, 2006, trad. fr., id., « Nous ne savions pas » : les Allemands et la Solution finale, 1933-1945, trad. de l'all. par Raymond Clarinard, Paris, Le Livre de poche, 2007, 2009; Ahlrich Meyer, Das Wissen um Auschwitz : Täter und Opfer der « Endlösung » in Westeuropa, Paderborn, Schöningh, 2010 ; Nicholas Stargardt, The German War : A Nation under Arms, 1939-1945, Londres, Bodley Head, 2015 ; trad. fr., id., La Guerre allemande : portrait d'un peuple en guerre, 1939-1945, trad. de l'angl. par Aude de Saint Loup et Pierre-Emmanuel Dauzat, Paris, Vuibert, 2017 ; Florent Brayard, «Those Who did not Believe: On the Reception of the Allied Declaration of December 17, 1942 », in Dina Porat et Dan Michman (dir.), The End of 1942: A Turning Point in World War II and in the Comprehension of the Final Solution?, Jerusalem, Yad Vashem, p. $117-133$.

(2) Archives nationales (AN, Pierrefitte-sur-Seine), Z6 146 n 2050, dossier d'instruction de Louis Sadosky. 
des témoignages, parfois contradictoires, lors du procès dans les années 1970 et confrontés au témoignage de 1942 d'un évadé du camp. À l'instar de l'ouvrage de Christopher Browning sur le camp de travail de Starachowice ${ }^{1}$, cet article illustre les transformations des positionnements des historiens et historiennes face aux témoignages. Essentiels dans la construction du savoir sur un grand nombre de lieux d'extermination dont il ne reste a priori aucune trace archivistique, ils sont bien souvent livrés après coup, ce qui n'est pas sans conséquence pour l'analyse. Le travail mené par Michael Pollak a montré combien ils donnaient d'abord à voir et à penser, bien plus que les trajectoires de la persécution, les modes et les caractéristiques de la survie ${ }^{2}$. Les récits ex post se construisent au cours d'interactions particulières et contextualisées, intégrant bien souvent la lumière de faits appris, lus ou redécouverts. Croiser les témoignages, sur un même fait, conduit à mettre en évidence leur plasticité ${ }^{3}$.

Il est certain que la disparition des témoins produit des effets quant aux manières d'observer les actes, de lire les textes, de décrire les gestes. La relation aux témoignages, contemporains ou non des événements, ego-documents ou rapports administratifs, textes ou documents audio ou audiovisuels, ne s'effectue plus dans le cadre d'interactions de visu. Le jeu avec les affects, qui traverse cette historiographie, en est modifié, que ce soit dans la relation des chercheurs aux acteurs, à l'objet de l'histoire ou au récit qui en est produit. Il ne s'agit pas ici de basculer vers une histoire des intimités, mais plutôt de retrouver un positionnement plus habituel de l'historien. La frontière entre fiction et histoire se défriche et se travaille, médiée par la source, dans ses différences formes, et non par la présence des témoins. Découvrir ces documents en l'absence de leurs auteurs et sans maitriser les questionnaires, les croiser avec d'autres sources produisent des effets contrastés, entre libération de l'emprise et fétichisation du vestige, qu'il est encore trop tôt pour décrire avec précision, mais qui vient placer la Shoah au rang d'une histoire dés-exceptionnalisée.

L'intégration de sources produites dans des contextes « hors Shoah » permet de rendre compte de processus à la lisière. Deux textes montrent l'intérêt de ce décentrement, à travers la focale des dossiers administratifs individuels. Antoine Burgard s'attache à rendre compte des mises en récit de la persécution à travers l'étude du millier de dossiers d'orphelins de la Shoah ayant immigré au Canada entre septembre 1947 et mars 1952. Il interroge cette source à l'aune de l'élaboration des savoirs historiens sur la Shoah en s'intéressant, plus précisément, aux manières dont les événements ont été vécus, dits, dépassés, oblitérés. Lire, en regard, la contribution de Marie-Bénédicte Vincent conduit à affronter la prise

(I) C. Browning, À l'intérieur d'un camp de travail nazi..., op. cit.

(2) Michael Pollak, L'Expérience concentrationnaire : essai sur le maintien de l'identité sociale, Paris, Métailié, 1990.

(3) Voir Rémy Besson, « La plasticité du témoignage : le cas Inge Deutschkron », in Magali Uhl (dir.), Les Récits visuels de soi : mises en récit artistiques et nouvelles scénographies de l'intime, Paris, Presses de Nanterre, 2015. 
en compte des crimes, dans l'immédiat après-guerre, interrogés dans leur dimension ordinaire. Elle entreprend en effet de comprendre les dits et non-dits via le dépouillement de centaines de procédures de contentieux déclenchées par d'anciens fonctionnaires de police, particulièrement impliqués dans les activités criminelles du régime nazi, afin d'obtenir la réintégration en République fédérale d'Allemagne de leurs anciens droits de fonctionnaires et de militaires de carrière. Cette source administrative permet de reconstituer les trajectoires de «fonctionnaires ordinaires » du nazisme, mais aussi d'interroger la nature des descriptions bureaucratiques, des agissements dans le cadre des procédures d'épuration.

\section{Pour un décloisonnement de l'histoire de la Shoah}

S'il est malaisé de prédire dans quel sens vont s'orienter les grandes questions des historiographies à venir, marquées par leurs propres présents et donc des avenirs incertains, il reste possible de dessiner quelques directions qui se dégagent de ces différents points de vue sur les sources. Un élargissement temporel, tout d'abord, qui conduit à inscrire la Shoah dans une périodisation étendue. Si l'on en reste à l'histoire des fonds documentaires, la périodisation doit être étendue à la première moitié du $20^{\mathrm{e}}$ siècle. Dirigée par Emanuel Ringelblum dans le ghetto de Varsovie, l'opération secrète Oyneg Shabes, de collecte systématique des traces des crimes perpétrés et des persécutions subies, traces de nature diverse (affiches, papiers officiels, décrets, photographies, dessins, témoignages, journaux intimes, tracts, etc.), fait référence à d'autres expériences de résistance via la constitution d'archives, qu'on pense aux travaux de Simon Doubnov dès la fin du $19^{\mathrm{e}}$ siècle sur l'histoire du judaïsme ou à ceux de l'Institut scientifique yiddish (YIVO) créé en 1925 en Pologne ${ }^{1}$. Il est indispensable d'y adjoindre l'après-guerre : une grande partie de ces archives, dissimulées sous terre en 1943 à l'intérieur de bidons de lait et de boîtes métalliques, est retrouvée en 1946 puis en 1950 à Varsovie, sous les décombres du ghetto. À Lodz, Nachman Zonabend, chargé par les Allemands de nettoyer les ruines du ghetto après sa liquidation, parvient à dissimuler une grande partie des archives, photographies et objets d'arts, qu'il donne en 1947 au YIVO². De même, en France, Isaac Schneersohn prend l'initiative à Grenoble, dès avril 1943, de lancer, avec une quarantaine de responsables de la communauté juive, une structure chargée de rassembler les preuves de la persécution dont ils sont victimes : le Centre de documentation juive contemporaine est né. Dans l'immédiat après-guerre, nombre d'initiatives équivalentes se multiplient parmi les survivants juifs ${ }^{3}$. Ces commissions historiques

(I) Samuel Kassow, Who Will Write Our History? Emanuel Ringelblum and the Oyneg Shabes Archive, Bloomington, Indiana University Press, 2007 ; trad. fr., id., Qui écrira notre histoire? Les archives secrètes du ghetto de Varsovie : Emanuel Ringelblum et les archives d'Oyneg Shabes, trad. de l'angl. par Pierre-Emmanuel Dauzat, Paris, Grasset, 2011, Flammarion, « Champs », 2013.

(2) Marek Web (dir.), The Documents of the Lodz Ghetto : An Inventory of the Nachman Zonabend Collection, New York, YIVO Institute for Jewish Research, 1988.

(3) Laura Jockusch, Collect and Record! Jewish Holocaust Documentation in Early Postwar Europe, New York, Oxford University Press, 2015. 
et centres de documentations s'attachent à rassembler d'importants ensembles archivistiques relatifs à « ce qui n'a encore pas pris le nom » de Shoah ou d'Holocauste ${ }^{1}$. Les articles de ce numéro témoignent de la vivacité des recherches sur l'après-guerre. L'élargissement chronologique ne consiste pas simplement à interroger la genèse et l'héritage des processus décrits ; il vise à donner une acception véritablement biographique à l'histoire de la Shoah. On peut ainsi rappeler le rôle, face à la persécution, des ressources sociales accumulées au cours des cheminements biographiques précédant l'événement, comme l'importance des trajectoires de persécution dans les parcours migratoires, professionnels ou matrimoniaux des survivants ${ }^{2}$. Inscrire l'extermination des juifs d'Europe dans une séquence élargie, du début des années 1920 aux années 1950, conduit ainsi à relire les rythmes de cette histoire à l'échelle non plus simplement des décideurs et des décisions, mais précisément des acteurs, en bref de lui redonner aussi une épaisseur biographique.

Une autre direction qui s'esquisse pourrait être qualifiée de distanciation à l'objet. L'interrogation sur les sources conduit les auteurs de ce numéro à adopter une posture critique vis-à-vis des documents exploités, à chaque fois resitués dans leurs contextes de production, leurs appropriations et leurs transmissions : pourquoi l'« album d'Auschwitz » s'est-il imposé dans les représentations de «l'irreprésentable » (Rachel Ertel) ? Quel rôle a joué la littérature dans la transmission? Rien d'exceptionnel dans ces positions, somme toute banales de l'historien. Néanmoins, l'unicité de la Shoah conjuguée à la quasi-omniprésence des acteurs, qu'ils soient vivants ou morts d'ailleurs, les rendaient parfois peu évidentes. La distance permise par le temps qui passe invite alors à penser une dimension centrale de l'histoire de la Shoah : le silence. Les déplacements de sources et les décalages de points de vue mettent en lumière ce qui est dit et ce qui est tu, ce qui est pensé et ce qui est nié, ce qui est vécu et ce qui est rêvé, ce dont on se rappelle et ce qu'on oublie. Une attention particulière est portée aux types d'énonciation et à leurs modalités : mots des orphelins pour raconter leurs expériences, angles des photographies, montages des films, contextes de témoignages, etc. Au cours de cette distanciation, la présence de l'historien dans l'histoire qu'il écrit reste encore bien souvent occultée ${ }^{3}$. Les non-dits, les esquives et les fugues que je continue moi-même de pratiquer s'accumulent. Ne serait-il pas plus judicieux pour notre génération, non pas aux fins de les dépasser mais plutôt de les intégrer à l'écriture de cette histoire, de simplement travailler sa relation à l'objet en l'explicitant ?

(I) Béla Rasky, Regina Fritz et Eva Kovács (dir.), Als der Holocaust noch keinen Namen hatte/Before the Holocaust Had Its Name. Zur frühen Aufarbeitung des NS-Massenmordes an den Fuden/Early Confrontations of the Nazi Mass Murder of the Jews, Vienne, New Academic Press, 2016.

(2) Pierre Mercklé et Claire Zalc, «Trajectories of the Persecuted During the Second World War: Contribution to a Microhistory of the Holocaust », in Philippe Blanchard, Felix Bühlmann et Jacques-Antoine Gauthier (dir.), Advances in Sequence Analysis : Theory, Method, Applications, New York, Springer, 2014, p. 171-190.

(3) Sauf lorsque l'historien entreprend l'histoire de sa propre famille, comme Ivan Jablonka, Histoire des grands-parents que je n'ai pas eus, op. cit. 
Enfin, j'aimerais espérer que ces différents mouvements se conjuguent pour œuvrer au décloisonnement de l'histoire de la Shoah. Annette Wieviorka a montré comment cette historiographie s'était construite en pans séparés, menés par deux types d'historiens distincts : d'une part l'histoire des bourreaux, menée par des historiens professionnels, ou plus exactement reconnus dans le milieu académique, d'autre part l'histoire des victimes, portée d'abord par les institutions juives et les historiens de la communauté juive ${ }^{1}$. Ce décloisonnement contribue, je crois, à lutter contre ce que certains qualifient de « séquestration de la Shoah » (David Engel), ou encore à dépasser le « mur » (Guy Miron) qui séparerait l'histoire de l'Holocauste de celle du monde juif ${ }^{2}$. Je sais que cet appel ne va pas dans le sens des logiques institutionnelles à l'œuvre aujourd'hui, de la spécialisation de l'histoire des génocides et de l'Holocauste (qui a désormais ses collections de livres dédiés, ses revues labellisées, ses instituts, un réseau de recherche européen) à ses possibilités particulières de financements : la Fondation pour la mémoire de la Shoah a ainsi délivré cent trente-sept allocations doctorales depuis 2003 et quarante-huit bourses postdoctorales, qui ne portent pas toutes d'ailleurs sur la Shoah (elle a accompagné le développement de l'histoire française du génocide rwandais). Cet exemple témoigne de l'essentielle et vitale diversité du champ, irrigué par des individus venus d'autres périodes et d'autres thématiques. Si l'on s'empare de cette histoire, c'est aussi pour y expérimenter des interrogations problématiques au long cours, qui s'y jouent avec une acuité particulière. La contribution d'Ivan Ermakoff, centrée sur un cas particulier de « ratage de l'obéissance programmée », vient alimenter une réflexion générale sur la contrainte et l'autonomie, les marges de manœuvre et les anticipations des acteurs face aux événements. L'histoire de la Shoah devient interrogée non dans sa spécificité, mais dans ce qu'elle engendre d'acquis de connaissances. En posant la question des sources de cette histoire, ce numéro teste les limites de la spécificité de cette historiographie aujourd'hui, en rappelant la pertinence des méthodologies habituelles du rapport aux sources, sur des terrains longtemps définis par leur exceptionnalité. Dès lors, il reste à en appeler à d'autres expérimentations historiennes (quantifications, spatialisations, analyses lexicales), comme à travailler l'ouverture avec les autres sciences sociales en renforçant le dialogue entre elles.

«Les sources du génocide, il faut le rappeler, restent des sources, et la recherche relative au génocide une recherche, écrit Raul Hilberg. Toute entreprise fondée sur des sources se révèle empirique et rien ne distingue à ces égards les travaux sur le génocide d'autres sujets

(I) A. Wieviorka, « Comprendre, témoigner, écrire », art. cité

(2) David Engel, Historians of the fews and the Holocaust, Stanford, Stanford University Press, 2009; Guy Miron, « Bridging the Divide : Holocaust versus Jewish History Research. Problems and Challenges », Yad Vashem Studies, 38 (2), 2010, p. 155-193. 
d'investigation [...]. La mise au jour constitue le but en soi, et le désir de comprendre ne connaît pas de limite ${ }^{1}$. »

\title{
Les jeux sont ouverts.
}

\author{
Claire Zalc, \\ École des hautes études en sciences sociales (EHESS), \\ Institut d'bistoire moderne et contemporaine (IHMC), \\ CNRS, 75005, Paris, France.
}

Claire Zalc est directrice de recherche au CNRS, à l'Institut d'histoire moderne et contemporaine (IHMC), et directrice d'études à l'École des hautes études en sciences sociales (EHESS). Ses recherches récentes croisent histoire des migrations et histoire des persécutions des juifs au $20^{\circledR}$ siècle. Elle a récemment publié Dénaturalisés : les retraits de nationalité sous Vichy (Éd. du Seuil, 2016). (claire.zalc@ens.fr)

(I) R. Hilberg, Les Sources de la Shoah..., op. cit., p. 199 et 261. Je remercie vivement pour leurs remarques sur ce texte Tal Bruttmann, Nicolas Mariot et Annette Wieviorka, ainsi que les membres du comité de Vingtième Siècle pour leurs commentaires au long de l'élaboration de ce numéro. Ce dernier n'aurait pu voir le jour sans l'aide de Virginie Durand. Qu'elle trouve ici l'expression de ma profonde gratitude. 